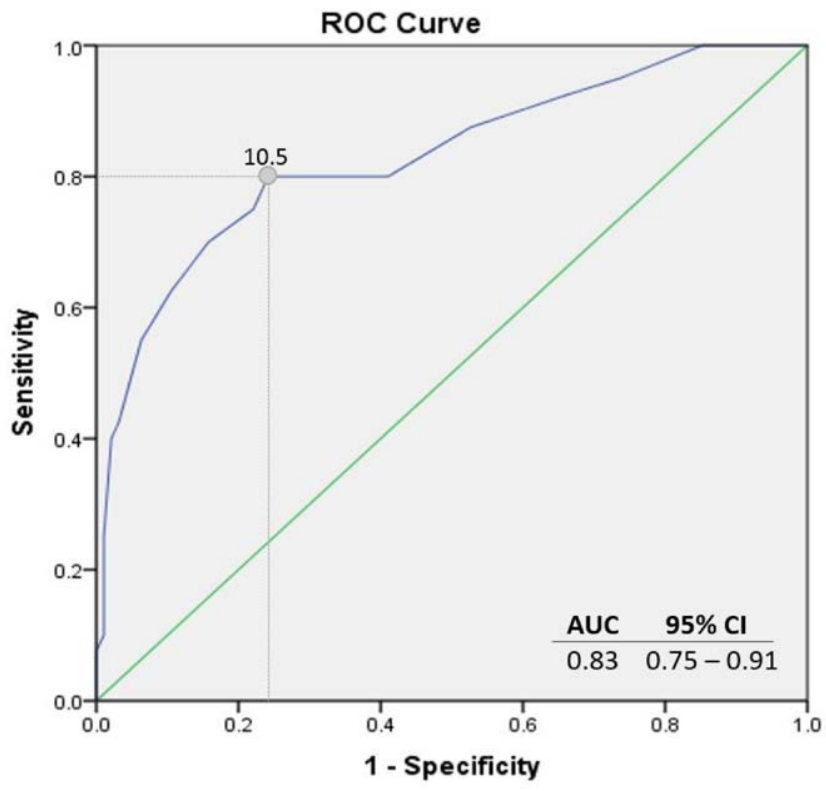

Figure 1. ROC curve depicting sensitivity and (1-) specificity. The AUC was $0.83(95.0 \% \mathrm{Cl}$ $0.75-0.91$ ) with an optimal cut-off value of 10.5 (sensitivity $80.0 \%$, specificity $75.8 \%$ ).

Disclosure of Interests: None declared DOI: 10.1136/annrheumdis-2021-eular.2466

\begin{tabular}{|l|l}
\hline AB0376 & LEVEL OF DIAGNOSTIC AND THERAPEUTIC \\
KNOWLEDGE, SKILLS AND COMPETENCIES IN GIANT \\
CELL ARTERITIS AMONG EMERGENCY MEDICAL \\
STAFF
\end{tabular}

C. Guillén-Astete $^{1}$, I. Perea-Fuentes ${ }^{2}$, L. Ayala-Terrados ${ }^{2}$, N. García-Montes ${ }^{3}$. ${ }^{1}$ Hospital Ramón y Cajal, Rheumatology, MADRID, Spain; ${ }^{2}$ CEU San Pablo, Medicine, Madrid, Spain; ${ }^{3}$ Hospital Ramón y Cajal, Emergency, Madrid, Spain

Background: Giant cell arteritis (GCA) is the most frequently diagnosed vasculitis in Spain. The highest incidence recorded in the country is 17 new cases per 100,000 inhabitants/year. Its delayed diagnosis exposes the patient to an increased risk of multiple ischaemic complications. Among them, vision loss has been reported in up to 1 in 6 cases. Most of these patients, according to unpublished local observations, presented with headache, visual disturbances or polymyalgic symptoms to an emergency department facility and were not correctly or prompted diagnosed.

Objectives: To determine the level of knowledge, skills and diagnostic and therapeutic competencies of the medical staff of multiple emergency departments in our city in regard to giant cell arteritis.

Methods: We designed an observational study based on a single survey carried out between February and March 2020 to medical staff from 12 emergency departments in the city of Madrid. Four private and eight public emergency departments were deliberately included. The selection of the surveyed staff was randomly selected to reach $50 \%$ of the total number of physicians in each department. A total of 229 surveys were distributed by e-mail. A total of 125 physicians responded (final response rate $54.5 \%$ ). Twenty-two physicians $(9.2 \%)$ did not respond to the request and the rest declined to participate, although they responded to the demographic section (35.8\%).

The survey included the following sections: respondent profile, knowledge of epidemiological aspects, identification of clinical manifestations, diagnostic and therapeutic skills, and educational interest.

Results: Forty respondents (33.6\%) worked in a privately managed emergency department and $79(66.4 \%)$ in a public centre. The mean age of respondents was 37.3 SD 5.9 years, and the proportion of women was $62.4 \%$. Mean length of service was 3.4 SD 1.2 years among physicians in private emergency departments and 5.7 SD 2.3 in a public management unit. Sixty-four respondents $(53.7 \%)$ were specialists in Family and Community Medicine, and 46 $(38.6 \%)$ in internal medicine. No significant differences in demographic characteristics were identified between respondents who agreed to participate and those who declined.
In regard to epidemiological knowledge, $50.6 \%$ of respondents were unable to identify the age group at highest risk of GCA, $32.7 \%$ did not recognise its predominance in the female sex, and $26 \%$ did not know the approximate incidence of the disease.

In terms of diagnostic suspicion, $46.2 \%$ of respondents were able to identify at least five clinical manifestations of the disease. Elevated ESR, CRP and the presence of anaemia were recognised as positive analytical data by $98.3 \%$, $85.7 \%$ and $57.7 \%$ of respondents, respectively. Ultrasonography of temporal arteries was identified by $97.5 \%$ of respondents as a useful diagnostic technique. One hundred and two respondents (81.6\%) indicated that they had not suspected GCA in the last month. In the last year, 34 respondents $(27.2 \%)$ reported having diagnosed the disease on at least one occasion. Regarding treatment, in cases of absence of ischaemic lesion $\mathbf{8 5 . 7 \%}$ indicated suboptimal corticosteroid therapy guidelines, however, $63.0 \%$ of the respondents self-rated their therapeutic skills as very good or excellent.

Finally, $88.2 \%$ of respondents expressed interest in participating in training programmes in diagnosis and treatment of GCA

Conclusion: There are important areas for improvement in knowledge, diagnostic skills and therapeutic competencies among emergency physicians in relation to the suspicion and management of GCA.

Disclosure of Interests: None declared

DOI: 10.1136/annrheumdis-2021-eular.2815

\section{AB0377 CLINICAL REVIEW OF 19 CASES WITH IDIOPATHIC AORTITIS - A SINGLE CENTRE STUDY}

M. J. Villar ${ }^{1}$, S. Sangle ${ }^{2}$, D. D'cruz ${ }^{2} .{ }^{1}$ Clinica Alemana, Reumatolog ía, Vitacura, Chile; ${ }^{2}$ Guys and St Thomas' Hospital NHS Foundation Trust, Louise Coote Lupus Unit, London, United Kingdom

Background: Aortitis is a group of disorders leading to inflammation in the aorta. When aortitis has no clinical evidence of systemic vasculitis, it is called idiopathic aortitis or clinically isolated aortitis (CIA).

There is no consensus on the management of CIA.

Objectives: Our purpose is to describe our cohort of patients with $\mathrm{CIA}$ and their response to treatment.

Methods: This is a retrospective analysis of 19 patients with CIA. All records of patients with $\mathrm{CIA}$ were analyzed and demographic variables, comorbidities, symptoms, images, histology, treatment

received, outcome and mortality were recorded. The description of quantitative variables was made using the median and the interquartile range (IQR).

Results: Nineteen patients were analyzed. Diagnosis was made by imaging in $18(94.7 \%)$, one patient was diagnosed by histology after aortic root surgery. Patient characteristics are detailed in Table 1.

The median duration of follow- up was 38 months (IQR 43). Seven patients $(36.8 \%)$ had constitutional symptoms including fever, weight loss, sweats and fatigue. $4(21.05 \%)$ presented with back or abdominal pain for a mean duration of 3 months (SD 0.81 ) before the diagnosis. In $8(42.1 \%)$ patients the diagnosis was made incidentally.

All patients had negative treponema, hepatitis $\mathrm{B}$, hepatitis $\mathrm{C}$ and Human Immunodeficiency Virus (HIV) serology.

All patients had negative autoimmune serology included ANCA. Interestingly $4(21.05 \%)$ had positive lupus anticoagulant without other manifestation of antiphospholipid syndrome.

The type of aortic involvement was aortitis in 10 patients $(52.6 \%)$, inflammatory aneurysm in $8(42.1 \%)$ and dissection in $1(5.2 \%)$.

Seven (36.8\%) patients had thoracic and abdominal aorta involvement, 6 (31.5\%) only thoracic aorta and 6 (31.5\%) only abdominal aorta.

Aortic histology was obtained in 5 patients, 2 had necrotizing arteritis with giant cell pattern and 2 had lymphoplasmacytic pattern. Temporal artery biopsy was done in one patient and the result was negative for GCA.

All patients received corticosteroids as a remission inducing agent, 12 $(63.1 \%)$ received methotrexate and $2(10.5 \%)$ mycophenolate. 2 patients died $(10.5 \%)$.

The median prednisone dose at the beginning was $20 \mathrm{mg}$ (IQR 20) and at remission was $5 \mathrm{mg}$ (IQR 20). 41.6\% (5/12) of patients treated with steroids plus methotrexate were able to stop steroids without reactivation over a median follow-up time of 23.5 months (IQR 31). (Table 2.)

Details of treatment, ESR and CRP pre and post treatment is shown in Figure 1.

Seven patients $(36.8 \%)$ had follow-up imaging, none of them showed active inflammation, new aneurysm, dissection or disease progression.

Conclusion: Treatment of isolated aortitis with steroids and methotrexate was effective resulting in clinical and laboratory improvement and allowed cessation or decrease in steroids to $5 \mathrm{mg}$ or less in $83 \%$ of patients. This therapeutic approach may be useful in patients with CIA. 